\title{
The nature of LINER galaxies: Ubiquitous hot old stars and rare accreting black holes
}

\author{
R. Singh, G. van de Ven, K. Jahnke, and the CALIFA collaboration
}

Max-Planck-Institut für Astronomie (MPIA), Königstuhl 17, 69117 Heidelberg, Germany

\begin{abstract}
Galaxies, which often contain ionised gas, sometimes also exhibit a so-called lowionisation nuclear emission line region (LINER). For 30 years, this was attributed to a central mass-accreting supermassive black hole (more commonly known as active galactic nucleus or AGN) of low luminosity, making LINER galaxies the largest AGN sub-population, which dominate in numbers over higher AGN-luminosity Seyfert galaxies and quasars. This, however, poses a serious problem. While the inferred energy balance is plausible, many LINERs clearly do not contain any other independent signatures of an AGN. Using integral field spectroscopic data from the CALIFA survey, we compare the observed radial surface brightness profiles with what is expected from illumination by an AGN. For 48 galaxies with LINER-like emission we show, that the radial emission-line surface brightness profiles are inconsistent with ionisation by a central point-source and hence cannot be due to an AGN alone. The most probable explanation for the excess LINER-like emission is ionisation by evolved stars during the short but very hot and energetic phase known as post-AGB. This leads us to an entirely new interpretation. Post-AGB stars are ubiquitous and their ionising effect should be potentially observable in every galaxy with gas present and with stars older than $\sim 1$ Gyr, unless a stronger radiation field from young hot stars or an AGN outshines them. This means, that galaxies with LINER-like emission are not a class defined by a property but rather by the absence of a property. It also explains why LINER emission is observed mostly in massive galaxies with old stars and little star formation.
\end{abstract}

Keywords. galaxies: active galaxies: ISM galaxies: nuclei stars: AGB and post-AGB

When LINERs were first identified as a class of galaxies in the early 1980s, it was clear that the necessary radiation field had to be different or have a different impact than for all previously known AGNs. Various explanations were put forward, ranging from shockionisation (Heckmann, 1980) via young hot stars (Terlevich et al., 1985) to the favoured ionisation by low-luminosity AGNs.

The latter explanation has strong implications, because LINERs make up most objects in the AGN class. While in the following decades the explanation that LINERs are powered by low-luminosity AGNs became generally accepted, doubts were again re-fuelled very recently. Inconsistencies were found between the AGN-ionisation hypothesis, and either predicted emission line strengths or the spatial distribution of LINER-like ionised regions in the galaxies, but neither were conclusive, because they either lacked full spatial or spectral information.

We test the picture of ionisation of the gas in these galaxies by an AGN, which predicts a radiation field declining with radius as $\propto 1 / r^{2}$. The Calar Alto Legacy Integral Field Area (CALIFA; Sánchez et al. 2012, Husemann 2012) survey provides the first dataset, with a substantial number of LINER galaxies with spatial resolution and the ability to spectrally identify LINER-like emission. The CALIFA survey is the first and ongoing IFS survey of a diameter-selected $\left(45<D_{25}<80\right)$ sample of up to 600 galaxies in the local universe $(0.005<z<0.03)$ of all Hubble types. The integral-field spectrograph PMAS/PPak mounted on the $3.5 \mathrm{~m}$ telescope at the Calar Alto has a field-of-view of $65 " \times 72$ " and covers the full optical extent of the selected galaxies. As described in Singh 

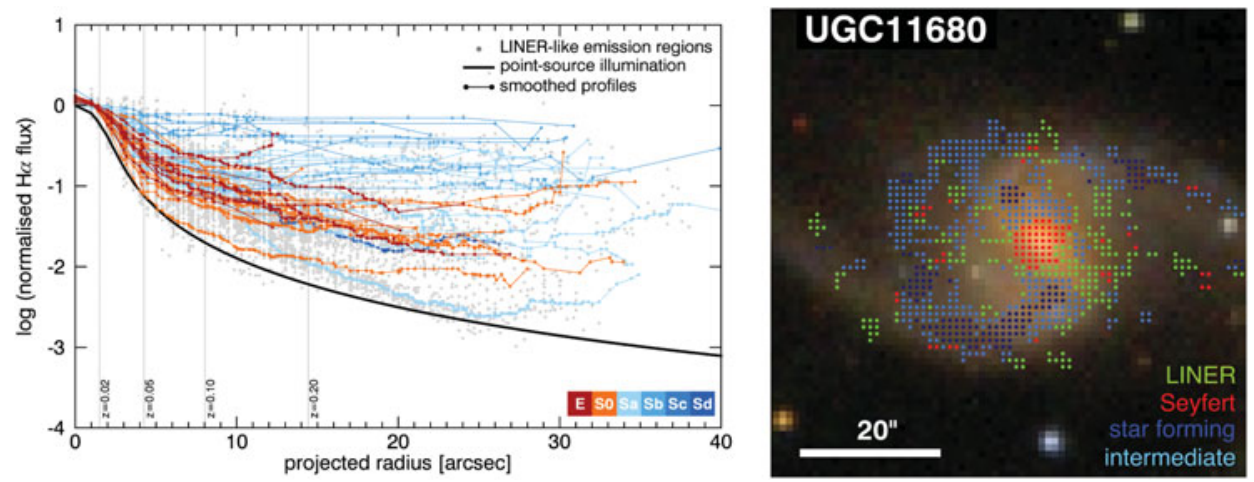

Figure 1: Left: flux profiles of LINER regions in 48 galaxies. Right: SDSS map with BPT overlay

et al. (2013), we devised a series of tests to ensure robustness of our results against projection effects, different emission line extraction methods, simplifying assumptions about the gas distribution and potential contamination of LINER regions by star formation. We showed that our results are robust against these tests.

In Fig. 1 (left) we selected only those regions with LINER-like emission from the 48 galaxies and plot the measured $\mathrm{H} \alpha$ surface brightness. After normalising the central $\mathrm{H} \alpha$ flux to unity for all galaxies, we arrive at the (smoothed) coloured radial profiles. The expected profile from central point-source illumination is plotted in black. There is a strong gap between the latter predicted point-source-illumination profile and the actual observed profiles. The vertical grey lines in Fig. 1 (left) illustrate the radial extent covered by a 3" diameter SDSS aperture, if the CALIFA galaxies with an average redshift of 0.017 would be placed at the indicated higher redshifts. This illustrates that SDSS emission line classification depends on distance and hence, apparent size.

Despite its name LINER-like emission covers all regions of the galaxies, as for example shown in Fig. 1 (right), aside from those regions where star-formation dominates the emission. The most probable energy source are hot evolved stars after their asymptotic giant branch (AGB) phase. This was already suggested before, but models of this phase in stellar evolution have only recently been picked up again (Stasińska 2008). This has the perplexing implication that every galaxy with stellar populations older than $\sim 1$ Gyr must have a radiation field from post-AGB stars that can ionise at least part of the interstellar gas when present. Even if LINER-like emission is predominantly powered by post-AGB stars, it does not preclude the existence of AGNs in LINER galaxies. The AGN could provide some of the central radiation in some LINER galaxies, but the LINER diagnostic is in general not a good predictor for the presence of an AGN.

\section{References}

Heckman, T. M. 1980, A\&A, 87, 152

Husemann, B., Jahnke, K., Sánchez, S. F., et al. 2013, A\&GA, 549, A87

Sánchez, S. F., Kennicutt, R. C., Gil de Paz, A., et al. 2012, A\&SA, 538, A8

Singh, R., Van de Ven, G., Jahnke, K., et al. 2013, A\&A, 558, A43

Stasińska, G., Vale Asari, N., Cid Fernandes, R., et al. 2008, MNRAS, 391, L29

Terlevich, R. \& Melnick, J. 1985, MNRAS, 213, 841 